\title{
Mutations of Fatty Acid Binding Protein (FABP) Genes of Arabidopsis Reduce Seed Formation and Affect Fatty Acid Composition
}

\author{
Micheline Ngankwimi Ngaki, Hilal Ilarslan and Eve Syrkin Wurtele \\ Department of Genetics, Development and Cell Biology, Iowa State University, Ames, IA \\ 50011
}

Fatty acids are physiologically critical as membrane constituents and fuel molecules, as well as for regulating protein functionality and intracellular signaling. Mechanisms that regulate fatty acid biosynthesis are not yet well understood. Bioinformatics analysis reveals three related genes of unknown function, herein called FABP genes, two of which are highly coexpressed within the fatty acid biosynthesis regulon (http://www.metnetdb.org) $[1,2]$.

Based on the expression pattern and sequence of the FABP genes, we postulated that they are involved in the regulation of fatty acid metabolism. To test this hypothesis, we generated transgenic plants harboring FABP promoter::GUS-GFP fusion constructs and found that FABP1, FABP2, FABP3 proteins are localized in plastids (Fig. 1), the site of de novo fatty acid biosynthesis in plant and the corresponding genes are most highly expressed in the cotyledons of young seedlings, siliques, seeds, developing embryos, and root tips (Fig. 2). The phenotype of fabpl lines was indistinguishable from wild type, however, in the fabp 2 plants, apical dominance is somewhat reduced. Furthermore, mutations of FABP genes have reduced seed formation and a significant proportion of aborted embryos. This aberrant seed development is most pronounced in fabp 2 mutants. Importantly, GC-MS analysis of leaves and seeds indicates fatty acid composition and level is altered in fabp mutants compared to wild type. Our data indicates the FABP genes are required for proper lipid metabolism.

\section{References}

[1] W. I. Mentzen and E. S. Wurtele, BMC Plant Biology 8 (99) (2008) 1-22; Sept 30 http://www.biomedcentral.com/1471-2229.

[2] W. I. Mentzen et al., BMC Plant Biology 8 (76), http://www.biomedcentral.com/14712229.

\section{Acknowledgements}

The microscopy was conducted at the Microscopy and NanoImaging Facility (MNIF) at Iowa State University. This research was funded by a grant from the National Science Foundation. 


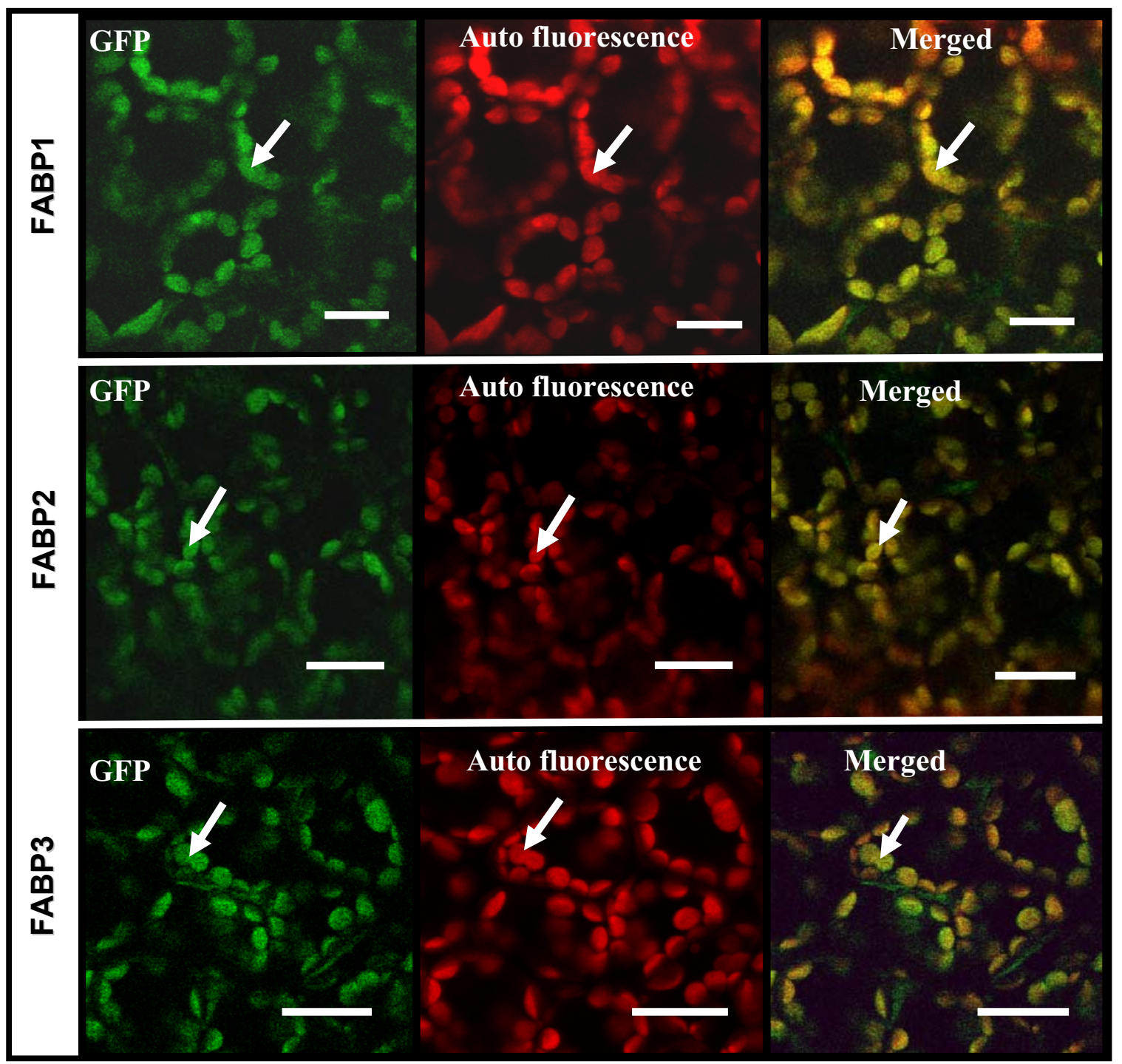

FIG. 1. FABP1, FABP2, FABP3 protein localized into chloroplasts. Bar scales $=20 \mu \mathrm{m}$.



FIG. 2. GUS expression patterns of FABP1, FABP2, FABP3 genes. 